\title{
Studien über die Veränderungen des Gewebseiweisses und dessen kolloid=osmotischen Drucks unter verschiedenen Bedingungen.
}

II. Gewebseiweiss und dessen kolloid-osmotischer Druck im Hunger.

\author{
Von \\ Shingo Yamamoto. \\ (山本新 吾) \\ (Aus der Medizinischen Klinik von Prof. T. Kato, \\ Tōhoku Reichsuniversität zu Sendai.)
}

Es ist vielfach darüber studiert worden, welche Veränderungen die Körper- u. Bluteiweisskörper im Hunger aufweisen (Blumentha ${ }^{1)}$ A bderhalden, Bergell u. Dörpinghaus, ${ }^{2)}$ Schoeneich, ${ }^{3}$ Morgulis u. Edwards, ${ }^{4)}$ Schelling, ${ }^{5)}$ Panum,, ${ }^{\text {() }}$ Burckhardt,") Robertson ${ }^{8}$ u.a.m.). Hatafuku u. Nakazawa ${ }^{9)}$ an hiesigerKlinik haben an Hunden das Verhalten von Eiweisskörpern und deren kolloidosmotischem Druck (k. o. D.) im Blut studiert und nachgewiesen, dass im Hunger der Eiweissgehalt des Blutes zunimmt, während der k. o.D. im kurzfristigen Hunger absinkt, im langfristigen Hunger ansteigt. Dies haben genannte Forscher auf die Zunahme der Eiweisskonzentration durch Bluteindickung sowie auf die Grössenveränderung der Eiweissteilchen durch Eiweisszersetzung zurückgeführt. Es erhebt sich nun die Frage, welche Veränderungen das Gewebseiweiss sowie der betreffende k.o.D. im Hunger aufweisen. Diese Frage ist für das

\footnotetext{
1) Blumentha], Dtsch. med. Wschr., 1903, 437.

2) Abderhalden, Bergell u. Dörpinghaus, Ztschr. f. physiol. Chem., 1904,

3) S choeneich, Ztschr. f. exp. Pathol. u. Therap., 1905-06, 2, 419.

4) Morgulis u. Ed wa rds, Amer. Journ. Physiol., 1924, 68, 477.

5) Schelling, Journ. Biol. Chem., 1930, 89, 575.

6) Pa n u m, Virchow's Arch., 1864, 29, 241.

7) Burckhardt, Arch. f. exp. Pathol u. Pharmakol, 1883, 16, 322.

8) Robertson, Journ. Biol. Chem., 1912-13, 13, 325.

9) Hatafuku u. Nakaz a wa, Tohoku. Journ. Exp. Med., 1933, 21, 226.
} 41, 153. 
Studium über den Einfluss des Hungers auf den Organismus von grossem Interesse, bleibt demnoch heute noch offen, deshalb habe ich vorliegende Versuche angestellt.

Versuchsmethodik: Man bringt gesunde ungefähr $2 \mathrm{~kg}$ schwere Kaninchen jedes für sich in den Käfig und lässt hungern. Der Hungerversuch wird in kurzfristigem auf 4 Tage, in langfristigem Versuch auf 7 Tage bemessen. Versuchstiere werden in 2 Gruppen geteilt, die eine Gruppe wird unter absoluter Karenz gehalten, die andere Gruppe bekommt nur Wasser nach Belieben. Die Bestimmungen des Eiweissgehaltes und dessen k.o.D. wurden, wie in der I. Mitteilung ${ }^{10)}$ einschlägiger Studien, am Leber-, Nieren- und Hirngewebe sowie am Blut ausgeführt.

Da die Bestimmungsmethode bereits in der I. Mitteilung geschildert wurde, mag ihre Beschreibung unterlassen werden.

Im übrigen sei einiges über ermittelte Werte erwähnt. Während bei Bestimmungen am Blut bei ein und demselben Tiere Werte vor und nach dem Hunger gegeneinander verglichen werden können, ist bei Bestimmungen an Hirn, Leber und Niere ein derartiges Verfahren nicht möglich. Aus diesem Grund habe ich unter Wiedergabe der in der I. Mitteilung bei normalen Kaninchen gewonnenen Durchschnittswerte einen Vergleich angestellt. In jeder Tabelle sind in Hinsicht darauf bei gesunden Kaninchen ermittelte Durchschnittswerte angegeben.

\section{Versuchsergebnisse in kurzfristigem Hunger.}

10 gesunde Kaninchen wurden in 2 Gruppen eingeteilt, die eine Gruppe wurde 4 Tage lang auf absolute Karenz gesetzt, während die andere Gruppe binnen gleicher Zeitdauer freiwillig Wasser erhielt. Versuchsergebnisse sind in Tab. 1 zusammengestellt. In dieser Tabelle sind unter der Gruppe A Werte, die an den unter absoluter Karenz gehaltenen Kaninchen gewonnen wurden, unter der Gruppe B Werte, die an Tieren, welche nach Belieben Wasser tranken, ermittelt wurden, aufgezeichnet.

Blut: Bei der Gruppe A, die auf absolute Karenz angewiesen wurde, wurde die Hämoglobinkonzentration ohne Ausnahme erhöht gefunden. Bei dieser Bluteindickung nahm Gesamt-N ab, Rest-N war vermehrt, so dass die Verminderung des Eiweiss-N auftrat. K. o.D. und Druck pro $100 \mathrm{mg} /$ dl Eiweiss-N sanken ebenfalls ab. Was die Werte bei der Gruppe B, der das Wassertrinken erlaubt war, anbelangt, erfuhr

10) Y a ma moto, Tohoku Journ. Exp. Med., 1938, 33, 342. 
Tabelle

Bestimungen bei

Gruppe A.

\begin{tabular}{|c|c|c|c|c|c|c|c|c|c|c|c|}
\hline \multirow[b]{2}{*}{ 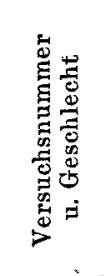 } & \multirow[b]{2}{*}{ 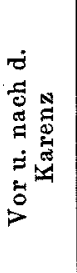 } & \multirow[b]{2}{*}{ 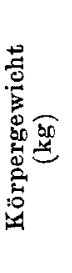 } & \multicolumn{6}{|c|}{ Blut } & \multicolumn{3}{|r|}{ Leber } \\
\hline & & & $\begin{array}{l}\frac{\widehat{\theta}}{8} \\
\dot{0} \\
\dot{0}\end{array}$ & 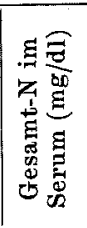 & 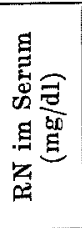 & 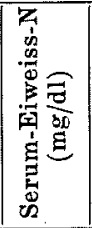 & 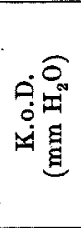 & 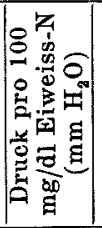 & 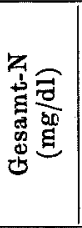 & 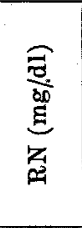 & 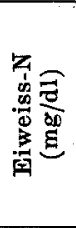 \\
\hline $\begin{array}{l}1 \\
\delta\end{array}$ & $\begin{array}{l}\text { Vor } \\
\text { Nach }\end{array}$ & $\begin{array}{l}1,540 \\
1,280\end{array}$ & $\begin{array}{l}14,06 \\
15,25\end{array}$ & $\begin{array}{l}978,6 \\
943,6\end{array}$ & $\begin{array}{l}29,5 \\
39,9\end{array}$ & $\begin{array}{l}947,1 \\
903,7\end{array}$ & $\begin{array}{l}305,0 \\
281,0\end{array}$ & $\begin{array}{l}32,2 \\
31,1\end{array}$ & 718,2 & 60,8 & 657,4 \\
\hline $\begin{array}{l}2 \\
5\end{array}$ & $\begin{array}{l}\text { Vor } \\
\text { Nach }\end{array}$ & $\begin{array}{l}1,700 \\
1,440\end{array}$ & $\begin{array}{l}14,52 \\
14,80\end{array}$ & $\begin{array}{r}1040,2 \\
971,6\end{array}$ & $\begin{array}{l}32,5 \\
38,8\end{array}$ & $\begin{array}{r}1007,7 \\
932,8\end{array}$ & $\begin{array}{l}339,0 \\
291,0\end{array}$ & $\begin{array}{l}34,6 \\
31,1\end{array}$ & 690,2 & 66,0 & $624 ; 2$ \\
\hline $\begin{array}{l}3 \\
9\end{array}$ & $\begin{array}{l}\text { Vor } \\
\text { Nach }\end{array}$ & $\begin{array}{l}1,890 \\
1,750\end{array}$ & $\begin{array}{l}14,03 \\
15,64\end{array}$ & $\begin{array}{l}999,6 \\
910,0\end{array}$ & $\begin{array}{l}40,8 \\
53,8\end{array}$ & $\begin{array}{l}958,8 \\
856,2\end{array}$ & $\begin{array}{l}299,0 \\
247,0\end{array}$ & $\begin{array}{l}30,3 \\
28,8\end{array}$ & 810,0 & 85,9 & 724,1 \\
\hline $\begin{array}{l}4 \\
8\end{array}$ & $\begin{array}{l}\text { Vor } \\
\text { Nach }\end{array}$ & $\begin{array}{l}1,900 \\
1,580\end{array}$ & $\begin{array}{l}11,66 \\
12,24\end{array}$ & $\begin{array}{r}1045,8 \\
905,8\end{array}$ & $\begin{array}{l}72,9 \\
87,7\end{array}$ & $\begin{array}{l}972,9 \\
818,1\end{array}$ & $\begin{array}{l}288,0 \\
223,0\end{array}$ & $\begin{array}{l}29,6 \\
27,3\end{array}$ & 762,4 & 106,8 & 655,6 \\
\hline $\begin{array}{l}5 \\
8\end{array}$ & $\begin{array}{l}\text { Vor } \\
\text { Nach }\end{array}$ & $\begin{array}{l}1,840 \\
1,330\end{array}$ & $\begin{array}{l}13,36 \\
14,32\end{array}$ & $\begin{array}{l}980,7 \\
917,1\end{array}$ & $\begin{array}{l}56,4 \\
73,8\end{array}$ & \begin{tabular}{|l|}
924,3 \\
843,3
\end{tabular} & $\begin{array}{l}299,0 \\
256,0\end{array}$ & $\begin{array}{l}32,3 \\
30,3\end{array}$ & 791,7 & 87,2 & 704,5 \\
\hline $\begin{array}{l}\text { Durch- } \\
\text { schnitt d } \\
\text { Differen }\end{array}$ & \multicolumn{2}{|c|}{$\begin{array}{l}\text { Vorwerte } \\
\text { Nachwerte } \\
\text { a } \%\end{array}$} & $\begin{array}{c}13,53 \\
14,45 \\
(+6,8 \\
\end{array}$ & $\begin{array}{r}1009,0 \\
929,6 \\
-7,9 \\
\end{array}$ & $\begin{array}{r}46,4 \\
58,8 \\
+26,7 \\
\end{array}$ & $\begin{array}{r}962,2 \\
870,8 \\
-0,5 \\
\end{array}$ & $\begin{array}{r}306,0 \\
259,6 \\
-11,2 \\
\end{array}$ & $\begin{array}{r}31,8 \\
29,7 \\
-6,6) \\
\end{array}$ & 754,5 & 81,3 & 673,2 \\
\hline \multicolumn{9}{|c|}{ Prozentische Ab-oder Zunahme im Vergleich mit d. Norm } & $-6,5$ & $+10,8$ & $-8,2$ \\
\hline
\end{tabular}

Gruppe B.

\begin{tabular}{|c|c|c|c|c|c|c|c|c|c|c|c|}
\hline $\begin{array}{l}1 \\
\text { 우 }\end{array}$ & $\begin{array}{l}\text { Vor } \\
\text { Nach }\end{array}$ & $\begin{array}{l}1,800 \\
1,520\end{array}$ & $\begin{array}{l}13,08 \\
13,20\end{array}$ & $\begin{array}{l}1099,0 \\
1009,4\end{array}$ & $\begin{array}{l}37,3 \\
45,1\end{array}$ & $\begin{array}{r}1061,7 \\
964,3\end{array}$ & $\begin{array}{l}299,0 \\
291,0\end{array}$ & $\begin{array}{l}28,2 \\
30,2\end{array}$ & 819,7 & 102,4 & 717,3 \\
\hline $\begin{array}{l}2 \\
\text { 우 }\end{array}$ & $\begin{array}{l}\text { Vor } \\
\text { Nach }\end{array}$ & $\begin{array}{l}1,700 \\
1,250\end{array}$ & $\begin{array}{l}11,08 \\
11,14\end{array}$ & $\begin{array}{l}924,0 \\
858,2\end{array}$ & $\begin{array}{l}36,5 \\
69,4\end{array}$ & $\begin{array}{l}887,5 \\
788,8\end{array}$ & $\begin{array}{l}339,0 \\
247,0\end{array}$ & $\begin{array}{l}38,2 \\
31,1\end{array}$ & 788,2 & 84,2 & 704,0 \\
\hline $\begin{array}{l}3 \\
\delta\end{array}$ & $\begin{array}{l}\text { Vor } \\
\text { Nach }\end{array}$ & $\begin{array}{l}2,170 \\
1,950\end{array}$ & $\begin{array}{l}12,94 \\
12,88\end{array}$ & $\begin{array}{l}931,0 \\
845,6\end{array}$ & $\begin{array}{l}47,7 \\
53,8\end{array}$ & $\begin{array}{l}883,8 \\
791,8\end{array}$ & $\begin{array}{l}319,0 \\
246,0\end{array}$ & $\begin{array}{l}36,1 \\
31,1\end{array}$ & 791,0 & 76,4 & 714,6 \\
\hline $\begin{array}{l}4 \\
\text { 우 }\end{array}$ & $\begin{array}{l}\text { Vor } \\
\text { Nach }\end{array}$ & $\begin{array}{l}1,670 \\
1,550\end{array}$ & $\begin{array}{l}11,75 \\
11,87\end{array}$ & $\begin{array}{r}1017,8 \\
959,0\end{array}$ & $\begin{array}{l}78,1 \\
98,0\end{array}$ & $\begin{array}{l}939,7 \\
861,0\end{array}$ & $\begin{array}{l}304,0 \\
262,0\end{array}$ & $\begin{array}{l}32,3 \\
30,4\end{array}$ & 700,0 & 85,9 & 614,1 \\
\hline $\begin{array}{l}5 \\
8\end{array}$ & $\begin{array}{l}\text { Vor } \\
\text { Nach }\end{array}$ & $\begin{array}{l}1,800 \\
1,520\end{array}$ & $\begin{array}{l}13,64 \\
13,70\end{array}$ & $\begin{array}{l}974,4 \\
966,0\end{array}$ & $\begin{array}{l}43,4 \\
46,8\end{array}$ & $\begin{array}{l}931,0 \\
919,2\end{array}$ & $\begin{array}{l}283,0 \\
250,0\end{array}$ & $\begin{array}{l}30,4 \\
27,2\end{array}$ & 751,8 & 87,2 & 664,6 \\
\hline $\begin{array}{l}\text { Durch- } \\
\text { schnittd. } \\
\text { Differenz } \\
\end{array}$ & \multicolumn{2}{|c|}{$\begin{array}{l}\text { Vorwerte } \\
\text { Nachwerte } \\
n \%\end{array}$} & \begin{tabular}{|}
12,50 \\
12,56 \\
$(+0,5$ \\
\end{tabular} & $\begin{array}{r}989,2 \\
927,6 \\
-6,6 \\
\end{array}$ & $\begin{array}{r}48,3 \\
62,7 \\
+29,8 \\
\end{array}$ & \begin{tabular}{|r|}
940,6 \\
865,0 \\
$-8,0$ \\
\end{tabular} & \begin{tabular}{|r|}
308,8 \\
259,2 \\
$-16,1$ \\
\end{tabular} & $\begin{array}{r}33,0 \\
30,0 \\
-9,1 \\
\end{array}$ & 770,1 & 87,2 & 682,9 \\
\hline \multicolumn{9}{|c|}{ Prozentische Ab-oder Zunahme im Vergleich mit d. Norm } & $-4,6$ & $+18,8$ & $-6,9$ \\
\hline \multicolumn{9}{|c|}{ Durchschnitt d. normalen Werte } & 806,9 & 73,4 & 733,5 \\
\hline
\end{tabular}


1.

kurzfristigem Hunger.

\begin{tabular}{|c|c|c|c|c|c|c|c|c|c|c|c|}
\hline \multicolumn{12}{|c|}{ (Absolute Karenz) } \\
\hline & & \multicolumn{5}{|c|}{ Niere } & \multicolumn{5}{|c|}{ Hirn } \\
\hline 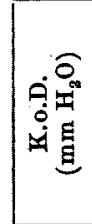 & 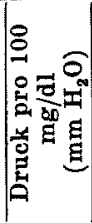 & 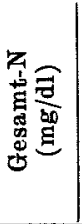 & 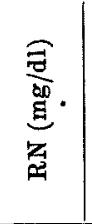 & 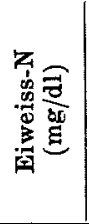 & 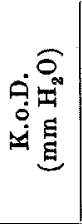 & 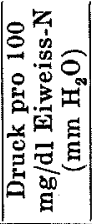 & 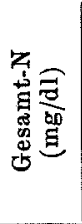 & 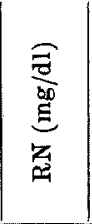 & 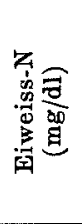 & 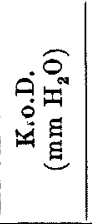 & 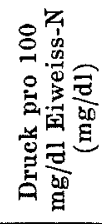 \\
\hline 111,0 & 16,9 & 571,2 & 81,6 & 489,6 & 80,0 & 16,3 & 225,4 & 47,7 & 177,7 & 15,0 & 8,4 \\
\hline 100,0 & 16,0 & 617,4 & 75,5 & 541,9 & 81,0 & 14,9 & 277,2 & 53,8 & 223,4 & 21,0 & $.9,4$ \\
\hline 115,0 & 15,9 & 603,4 & 96,3 & 507,1 & 81,0 & 16,0 & 267,4 & 80,7 & 186,7 & 18,0 & 9,6 \\
\hline 107,0 & 16,3 & 614,6 & 95,9 & 518,7 & 82,0 & 15,8 & 224,0 & 50,1 & 173,9 & 16,0 & 9,4 \\
\hline 102,0 & 14,5 & 509,4 & 105,9 & 403,5 & 68,0 & $.16,8$ & 259,0 & 54,7 & 204,3 & 18,0 & 8,8 \\
\hline 107,0 & 15,9 & 583,2 & 91,0 & 492,2 & $\mathbf{7 8 , 4}$ & 16,0 & 250,6 & 57,4 & 193,2 & 17,6 & 9,1 \\
\hline$-7,9$ & \pm 0 & $-1,4$ & $+32,8$ & $-5,9$ & $-3,1$ & $+2,6$ & $+8,2$ & $+44,6$ & $+0,7$ & $--12,9$ & $-13,3$ \\
\hline
\end{tabular}

(Trinkwasser nach Belieben)

\begin{tabular}{|c|c|c|c|c|c|c|c|c|c|c|c|}
\hline 100,0 & 13,9 & 602,0 & 118,5 & 483,5 & 98,0 & 20,2 & 275,8 & 44,3 & 231,5 & 15,0 & 6,5 \\
\hline 115,0 & 16,3 & 522,2 & 79,0 & 443,2 & 81,0 & 18,3 & 180,6 & 45,1 & 135,5 & 14,0 & 10,3 \\
\hline 109,0 & 15,2 & 560,0 & 99,8 & 460,2 & 80,0 & 17,3 & 233,8 & 42,5 & 191,3 & 17,0 & 8,9 \\
\hline 90,0 & 14,6 & 530,6 & 42,5 & 488,1 & 74,0 & 15,2 & 238,0 & 45,1 & 192,9 & 17,0 & 8,8 \\
\hline 102,0 & 15,3 & 623,0 & 105,9 & 517,1 & 74,0 & 14,3 & 245,0 & 54,7 & 190,3 & 18,0 & 9,4 \\
\hline 103,2 & 15,1 & 567,6 & 89,1 & 478,4 & 81,4 & 17,1 & 234,6 & 46,3 & 188,3 & 16,2 & 8,8 \\
\hline$-11,2$ & $-5,0$ & $-4,1$ & $+30,1$ & $-8,6$ & $+6,2$ & $+9,6$ & $+1,3$ & $+16,6$ & $-1,8$ & $-19,8$ & $-16,2$ \\
\hline 116,2 & 15,9 & 591,7 & 68,5 & 523,2 & 80,9 & 15,6 & 231,6 & 39,7 & 191,8 & 20,2 & 10,5 \\
\hline
\end{tabular}


Tabelle

Bestimmungèn bei

\begin{tabular}{|c|c|c|c|c|c|c|c|c|c|c|c|}
\hline & & & & & & & & & & \multicolumn{2}{|c|}{ Gruppe A. } \\
\hline \multirow[b]{2}{*}{ 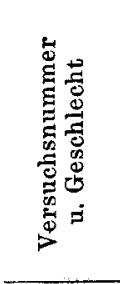 } & \multirow[b]{2}{*}{ 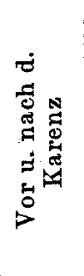 } & \multirow[b]{2}{*}{ 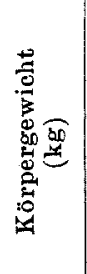 } & \multicolumn{6}{|c|}{ Blut } & \multicolumn{3}{|r|}{ Leber } \\
\hline & & & $\underbrace{\frac{\hat{\theta}}{\partial x}}_{\hat{I}}$ & 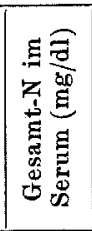 & 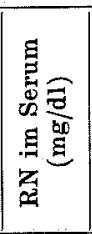 & 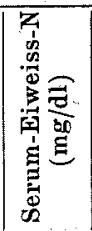 & 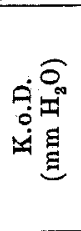 & 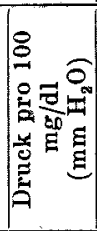 & 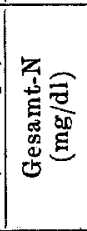 & 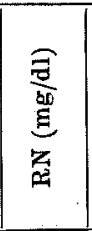 & 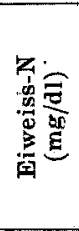 \\
\hline $\begin{array}{l}1 \\
8\end{array}$ & $\begin{array}{l}\text { Vor } \\
\text { Nach }\end{array}$ & $\begin{array}{l}2,470 \\
1,790\end{array}$ & $\begin{array}{l}12,38 \\
14,00\end{array}$ & $\begin{array}{l}1278,2 \\
1384,5\end{array}$ & $\begin{array}{r}56,9 \\
105,0\end{array}$ & $\begin{array}{l}1221,3 \\
1279,5\end{array}$ & $\begin{array}{l}376,5 \\
361,0\end{array}$ & $\begin{array}{l}30,8 \\
28,2\end{array}$ & 854,0 & 97,2 & 756,8 \\
\hline $\begin{array}{l}2 \\
q\end{array}$ & $\begin{array}{l}\text { Vor } \\
\text { Nach }\end{array}$ & $\begin{array}{l}2,500 \\
1,840\end{array}$ & $\begin{array}{l}13,53 \\
13,75\end{array}$ & $\begin{array}{l}1119,0 \\
1093,5\end{array}$ & $\begin{array}{l}33,0 \\
\mathbf{9 3}, 8\end{array}$ & $\begin{array}{r}1086,0 \\
999,7\end{array}$ & $\begin{array}{l}350,0 \\
298,0\end{array}$ & $\begin{array}{l}32,2 \\
29,8\end{array}$ & 1014,0 & 100,7 & 913,3 \\
\hline $\begin{array}{l}3 \\
\dot{+}\end{array}$ & $\begin{array}{l}\text { Vor } \\
\text { Nach }\end{array}$ & $\begin{array}{l}2,700 \\
1,560\end{array}$ & $\begin{array}{l}13,64 \\
14,83\end{array}$ & $\begin{array}{l}1281,0 \\
1047,2\end{array}$ & $\begin{array}{l}43,8 \\
80,3\end{array}$ & $\begin{array}{r}1237,2 \\
964,2\end{array}$ & $\begin{array}{l}320,0 \\
287,0\end{array}$ & $\begin{array}{l}25,9 \\
29,8\end{array}$ & 858,2 & 87,2 & 771,0 \\
\hline $\begin{array}{l}4 \\
\text { 今 }\end{array}$ & $\begin{array}{l}\text { Vor } \\
\text { Nach }\end{array}$ & $\begin{array}{l}2,780 \\
1,740\end{array}$ & $\begin{array}{l}13,36 \\
14,38\end{array}$ & $\begin{array}{r}971,6 \\
1141,0\end{array}$ & $\begin{array}{l}50,0 \\
90,3\end{array}$ & $\begin{array}{r}921,6 \\
1050,7\end{array}$ & $\begin{array}{r}266,5 \\
337,0\end{array}$ & $\begin{array}{l}28,9 \\
32,1\end{array}$ & 827,4 & 91,1 & 736,3 \\
\hline $\begin{array}{l}\mathbf{5} \\
8\end{array}$ & $\begin{array}{l}\text { Vor } \\
\text { Nach }\end{array}$ & $\begin{array}{l}2,470 \\
1,380\end{array}$ & $\begin{array}{l}13,76 \\
14,80\end{array}$ & $\begin{array}{l}926,9 \\
981,5\end{array}$ & $\begin{array}{l}48,6 \\
92,9\end{array}$ & $\begin{array}{l}878,3 \\
888,6\end{array}$ & $\begin{array}{l}292,5 \\
280,0\end{array}$ & $\begin{array}{l}38,3 \\
31,5\end{array}$ & 889,4 & 95,3 & 794,1 \\
\hline $\begin{array}{l}\text { Durch- } \\
\text { schnitt d. } \\
\text { Differenz }\end{array}$ & \multicolumn{2}{|c|}{$\begin{array}{l}\text { Vorwerte } \\
\text { Nachwerte } \\
\text { n } \%\end{array}$} & $\begin{array}{c}13,33 \\
14,35 \\
+7,6\end{array}$ & $\begin{array}{r}1115,3 \\
1129,5 \\
+1,3\end{array}$ & $\begin{array}{r}46,5 \\
92,5 \\
+98,9 \\
\end{array}$ & \begin{tabular}{|r|}
1068,9 \\
1036,5 \\
$-3,0$
\end{tabular} & $\begin{array}{l}321,1 \\
312,6 \\
-2,6\end{array}$ & $\begin{array}{r}30,2 \\
30,3 \\
+0,3) \\
\end{array}$ & 888,6 & 94,3 & 794,3 \\
\hline \multicolumn{9}{|c|}{ Prozentische Ab- oder Zunahme im Vergleich mit d. Norm } & $+10,1$ & $+28,5$ & $+8,3$ \\
\hline
\end{tabular}

Gruppe B.

\begin{tabular}{|c|c|c|c|c|c|c|c|c|c|c|c|}
\hline $\begin{array}{l}1 \\
\text { 우 }\end{array}$ & $\begin{array}{l}\text { Vor } \\
\text { Nach }\end{array}$ & $\begin{array}{l}2,380 \\
1,230\end{array}$ & $\begin{array}{l}13,50 \\
13,67\end{array}$ & $\begin{array}{r}1018,5 \\
973,0\end{array}$ & $\begin{array}{l}27,8 \\
61,2\end{array}$ & $\begin{array}{l}990,7 \\
911,2\end{array}$ & $\begin{array}{l}321,4 \\
270,0\end{array}$ & $\begin{array}{l}32,4 \\
29,6\end{array}$ & 896,7 & 83,3 & 813,4 \\
\hline $\begin{array}{l}2 \\
\text { 今 }\end{array}$ & $\begin{array}{l}\text { Vor } \\
\text { Nach }\end{array}$ & $\begin{array}{l}2,000 \\
1,490\end{array}$ & $\begin{array}{l}14,21 \\
14,35\end{array}$ & $\begin{array}{l}934,5 \\
971,6\end{array}$ & $\begin{array}{l}36,5 \\
87,7\end{array}$ & $\begin{array}{l}898,0 \\
883,9\end{array}$ & $\begin{array}{l}278,5 \\
280,0\end{array}$ & $\begin{array}{l}31,0 \\
31,7\end{array}$ & 779,8 & 105,9 & 673,9 \\
\hline $\begin{array}{l}3 \\
\text { 우 }\end{array}$ & $\begin{array}{l}\text { Vor } \\
\text { Nach }\end{array}$ & $\begin{array}{l}2,260 \\
1,650\end{array}$ & $\begin{array}{l}12,80 \\
13,22\end{array}$ & $\begin{array}{r}922,6 \\
1012,2\end{array}$ & $\begin{array}{l}45,1 \\
66,8\end{array}$ & $\begin{array}{l}877,5 \\
945,4\end{array}$ & $\begin{array}{l}298,0 \\
331,5\end{array}$ & $\begin{array}{l}34,0 \\
35,1\end{array}$ & 760,2 & 95,9 & 664,3 \\
\hline $\begin{array}{l}4 \\
\text { 今 }\end{array}$ & $\begin{array}{l}\text { Vor } \\
\text { Nach }\end{array}$ & $\begin{array}{l}2,000 \\
1,490\end{array}$ & $\begin{array}{l}14,83 \\
15,36\end{array}$ & $\begin{array}{l}977,2 \\
873,6\end{array}$ & $\begin{array}{l}31,2 \\
70,3\end{array}$ & $\begin{array}{l}946,0 \\
803,3\end{array}$ & $\begin{array}{l}368,0 \\
348,0\end{array}$ & $\begin{array}{l}38,9 \\
43,3\end{array}$ & 840,7 & 93,7 & $\mathbf{7 4 7 , 0}$ \\
\hline $\begin{array}{l}5 \\
\delta\end{array}$ & $\begin{array}{l}\text { Vor } \\
\text { Nach }\end{array}$ & $\begin{array}{l}2,350 \\
1,820\end{array}$ & $\begin{array}{l}14,06 \\
14,52\end{array}$ & $\begin{array}{l}936,6 \\
974,4\end{array}$ & $\begin{array}{l}44,3 \\
58,2\end{array}$ & $\begin{array}{l}892,3 \\
916,2\end{array}$ & $\begin{array}{l}297,0 \\
280,0\end{array}$ & $\begin{array}{l}33,3 \\
30,6\end{array}$ & 871,6 & 89,4 & 782,2 \\
\hline $\begin{array}{l}\text { Durch- } \\
\text { schnitt d. } \\
\text { Differenz }\end{array}$ & \multicolumn{2}{|c|}{$\begin{array}{l}\text { Vorwerte } \\
\text { Nachwerte } \\
n \%\end{array}$} & $\begin{array}{c}33,88 \\
44,22 \\
(+2,4\end{array}$ & $\begin{array}{l}957,9 \\
961,0 \\
+0,3\end{array}$ & $\begin{array}{r}37,0 \\
68,8 \\
+85,9 \\
\end{array}$ & $\begin{array}{l}920,9 \\
892,2 \\
-3,1\end{array}$ & $\begin{array}{r}312,6 \\
302,0 \\
-3,4\end{array}$ & $\begin{array}{r}33,9 \\
34,1 \\
+0,6) \\
\end{array}$ & 829,8 & 93,6 & $\mathbf{7 3 6 , 2}$ \\
\hline \multicolumn{9}{|c|}{ Prozentische Ab-oder Zunahme im Vergleich mit d. Norm } & $+2,8$ & $+27,5$ & $+0,4$ \\
\hline \multicolumn{9}{|c|}{ Durchschnitt d. normalen Werte } & 806,9 & 73,4 & 733,5 \\
\hline
\end{tabular}


2.

langfristigem Hunger.

\begin{tabular}{|c|c|c|c|c|c|c|c|c|c|c|c|}
\hline \multicolumn{12}{|c|}{ (Absolute Karenz) } \\
\hline & & \multicolumn{5}{|c|}{ Niere } & \multicolumn{5}{|c|}{ Hirn } \\
\hline 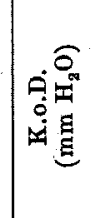 & 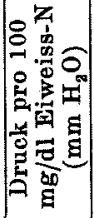 & 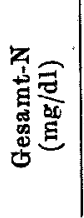 & 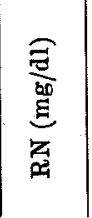 & 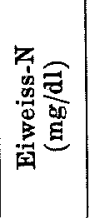 & 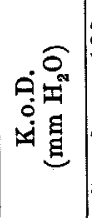 & 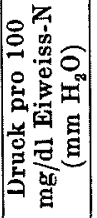 & 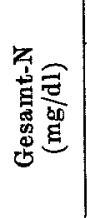 & 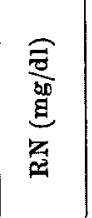 & 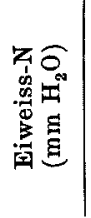 & 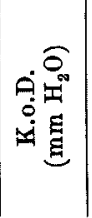 & 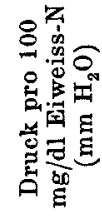 \\
\hline 107,0 & 14,1 & 585,2 & 128,5 & 456,7 & 76,0 & 16,0 & 240,0 & 66,0 & 174,0 & 13,0 & 7,5 \\
\hline 101,0 & 11,0 & 607,5 & 108,6 & 498,9 & 70,0 & 14,0 & 273,0 & 59,1 & 213,9 & 13,0 & 6,1 \\
\hline 109,0 & 14,1 & 651,0 & 110,7 & 540,3 & 89,0 & 16,4 & 238,0 & 58,2 & 179,8 & 18,0 & 8,0 \\
\hline $\mathbf{9 8 , 0}$ & 13,3 & 586,6 & 126,7 & 459,9 & 67,0 & 14,5 & 210,7 & 79,9 & 130,8 & 15,0 & 11,5 \\
\hline 89,0 & 11,5 & 572,4 & 119,4 & 453,0 & 61,0 & 13,4 & 228,4 & 63,2 & 165,2 & 17,0 & 10,3 \\
\hline 100,8 & 12,8 & 600,5 & 118,8 & 481,8 & 72,6 & 15,0 & 238,0 & 65,3 & 172,7 & 15,2 & 8,7 \\
\hline$-13,2$ & $-19,5$ & $+1,5$ & $+73,4$ & $-7,9$ & $-10,2$ & $-3,8$ & $+2,8$ & $+64,5$ & $-10,0$ & $-24,7$ & $-17,1$ \\
\hline \multicolumn{12}{|c|}{ (Trinkwasser nach Belieben) } \\
\hline 97,0 & 11,9 & 642,6 & 83,3 & 559,3 & 60,0 & 10,7 & 261,8 & 55,5 & 206,3 & 12,0 & 5,8 \\
\hline 96,0 & 14,2 & 622,3 & 91,1 & 531,2 & 63,0 & 11,7 & 226,1 & 43,4 & 182,7 & 11,0 & 6,0 \\
\hline 98,0 & 14,7 & 674,1 & 55,5 & 618,5 & 77,0 & 12,4 & 312,2 & 52,1 & 260,1 & 18,0 & 6,9 \\
\hline 104,0 & 13,9 & 641,2 & 111,5 & 529,7 & 74,0 & 14,0 & 219,2 & 64,2 & 155,0 & 12,0 & 7,7 \\
\hline 112,0 & 12,7 & 555,8 & 89,4 & 466,4 & 68,0 & 14,6 & 246,4 & 60,8 & 185,6 & 13,0 & 7,0 \\
\hline 101,4 & 13,5 & 627,2 & 86,2 & 541,0 & 68,4 & 12,7 & 253,1 & 55,2 & 197,9 & 13,2 & 6,7 \\
\hline$-12,7$ & $-15,1$ & $+6,0$ & $+25,8$ & $+3,4$ & $-15,4$ & $-18,6$ & $+9,3$ & $+39,0$ & $+3,2$ & $-34,6$ & $-36,2$ \\
\hline 116,2 & 15,9 & 591,7 & 68,5 & 523,2 & 80,9 & 15,6 & 231,6 & 39,7 & 191,8 & 20,2 & 10,5 \\
\hline
\end{tabular}


die Hämoglobinkonzentration fast keine Änderung. Bei diesem Konstantbleiben der Blutkonzentration nahm ebenfalls wie bei der Gruppe A Gesamt-N ab, Rest-N zu und Eiweiss-N ab. K.o.D. und Druck pro $100 \mathrm{mg} /$ dl Eiweiss-N fielen ab. Aus oben angeführtem Ergebnisse geht klar hervor, dass in bezug auf das Blut die Wasserverabreichung nur den Unterschied in der Hämoglobinkonzentration hervorruft, auf das Verhalten von Eiweisskörpern und deren k.o.D. aber keinen Einfluss ausübt.

Gewebe : Im Hirn nahm Rest- $\mathrm{N}$ bei beliebiger Wasseraufnahme gewissermassen zu, trotzdem Gesamt- $\mathrm{N}$ und Eiweiss- $\mathrm{N}$ an annähernd normalen Grenzen lagen. Bei absoluter Karenz war Gesamt-N zwar erhöht, aber wegen weitgehend erheblicher Zunahme des Rest-N lag Eiweiss-N im Bereich des annähernden normalen Wertes. K.o.D. sowie Druck pro $100 \mathrm{mg} / \mathrm{dl}$ Eiweiss-N sanken jedenfalls ab, gleichviel, ob Wasser gegeben wurde oder nicht.

In der Leber war Gesamt- $\mathrm{N}$ unter jeder Bedingung vermindert, Rest-N war deutlich vermehrt, so dass Eiweiss-N abnahm. Da k.o.D. mit der Abnahme des Eiweiss-N beinahe parallel abfiel, wies Druck pro $100 \mathrm{mg} /$ dl Eiweiss-N keine besondere Veränderung auf. In der Niere nahm Gesamt-N unter beiden Umständen einigermassen $a b$; weil jedoch Rest- $\mathrm{N}$ ausgeprägt erhöht war, nahm Eiweiss-N ab. Da k. o. D. trotz der Abnahme des Eiweiss-N nahe am normalen Wert lag, zeigte Druck pro $100 \mathrm{mg} /$ dl Eiweiss-N die Tendenz zu mässiger Erhöhung.

\section{Versuchsergebnisse bei langfristigem Hunger.}

Ebenso wie bei vorheriger Versuchsanordnung wurden 10 Kaninchen in 2 Gruppen eingeteilt, die eine Gruppe wurde 7 Tage lang auf absolute Karenz gesetzt, während die andere während gleichen Zeitraumes nur Wasser allein nach Belieben bekam. Nach 7tägigem Hungerversuch wurden Bestimmungen ausgeführt. Diese Resultate sind in Tab. 2 zusammengestellt. In der Tabelle sind unter der Gruppe A Werte bei Tieren, die auf absolute Karenz gesetzt wurden, unter der Gruppe B Werte bei Tieren, die nur Wasser allein nach Belieben zu sich nahmen, aufgezeichnet.

Bezüglich der Verhältnisse im Blut nahm die Hämoglobinkonzentration bei absoluter Karenz deutich zu. Auch in den Fällen, wo die Wasseraufnahme erlaubt war, war die Hämoglobinkonzentration mehr oder weniger erhöht. Das Blut war also bei absoluter Karenz deut- 
lich, bei Wasserzufuhr in geringem Masse eingedickt.

Gesamt- $N$ erfuhr, den einzelnen Versuchen nach betrachtet, sowohl bei der Gruppe A wie auch bei der Gruppe B in 3 von 5 Versuchen eine geringe Zunahme, in übrigen 2 Versuchen eine geringe Abnahme. Im ganzen genommen aber lässt sich sagen, dass Gesamt-N keine nennenswerte Schwankung aufweist, weil er jedenfalls innerhalb der normalen Grenzen schwankte. Rest-N wurde unter beiden Bedingungen deutlich vermehrt gefunden. Im besonderen bei absoluter Karenz wurde erheblichere Zunahme angetroffen. Eiweiss-N nahm bei beiden Tiergruppen ab. K.o.D. sank auch allemal im Verein mit der Abnahme des Eiweiss-N ab. Druck pro $100 \mathrm{mg} / \mathrm{dl}$ Eiweiss-N zeigte keine bemerkbare Schwankung.

Im,Hirngewebe zeigte Gesamt-N bei absoluter Karenz annähernde Normalwerte, bei Wasseraufnahme eine mehr oder weniger Zunahme. Rest-N nahm bei beiden Gruppen zu, besonders ausgeprägt war diese Zunahme bei absoluter Karenz. Folglich nahm Eiweiss-N bei absoluter Karenz deutlich ab, während er bei beliebiger Wasseraufnahme fast keine Schwankung aufwies. K.o.D. und Druck pro $100 \mathrm{mg} / \mathrm{dl}$ Eiweiss- $\mathrm{N}$ fielen bei beiden Gruppen ab, und zwar war dieser Abfall bei Wasseraufnahme etwas höhergradig.

In der Leber nahm Gesamt-N unabhängig von der Wasserzufuhr zu, der Prozentsatz dieser Zunahme war bei absoluter Karenz stärker. Rest- $N$ zeigte beinahe gleichgradige Erhöhung bei beiden Gruppen. Eiweiss- $\mathrm{N}$ zeigte bei Wasseraufnahme annähernde Normalwerte, wurde aber bei absoluter Karenz vermehrt gefunden. K. o. D. sowie Druck pro $100 \mathrm{mg} / \mathrm{dl}$ Eiweiss-N fielen unter beiden Bedingungen mehr oder weniger $a b$.

In der Niere erfuhr Gesamt- $\mathrm{N}$ bei Wasserzufuhr eine geringe $\mathrm{Zu}$ nahme, bei absolutem Hunger fast keine Schwankung; Rest-N war bei beiden Gruppen beträchtlich vermehrt, bei absolutem Hunger in besonders stärkerem Masse. Eiweiss-N nahm bei absoluter Karenz ab, bei Wasseraufnahme aber um ein Geringes zu. K.o.D. sowie Druck pro $100 \mathrm{mg} / \mathrm{dl}$ Eiweiss-N waren bei Wasseraufnahme erniedrigt, bei absoluter Karenz sank der k.o.D. ein wenig ab, wobei Druck pro 100 $\mathrm{mg} / \mathrm{dl}$ Eiweiss-N fast keine Schwankung aufwies.

\section{Besprechung.}

Ưber diesbezügliche Veränderungen im Blut liegen bereits die Untersuchungen von $\mathrm{Hatafuku} u$. Nakazawa ${ }^{9)}$ an hiesiger Klinik 
vor; genannte Forscher haben Experiment an Hunden angestellt, während meine Versuche an Kaninchen ausgeführt wurden. Es kommt hierbei nicht zuletzt darauf an, auf etwa vorkommende Unterschiede in Ergebnissen durch Verschiedenheit des Versuchsobjektes zu prüfen. Denn Roberts on ${ }^{8)}$ hat den Beweis geführt, dass die Veränderung des Bluteiweisses durch Hunger sich je nach der Art der Versuchstiere nicht immer gleichsinnig verhält.

In erster Linie nimmt das Hämoglobin im Hunger an Konzentration zu, was bei absoluter Karenz besonders auffallend auftritt. Bei Tieren, die Wasser genommen haben, ist die Hämoglobinkonzentration im kurzfristigen Versuch kaum erhöht, im langfristigen Versuch aber in allen Versuchen gesteigert. Dass bei Wasserzufuhr die Erhöhung der Hämoglobinkonzentration leichtgradig ist, ist selbstverständlich, und zwar gleichviel, ob es sich um Hund oder" um Kaninchen handelt.

Bevor man über Veränderungen an Eiweisskörpern diskutiert, muss man zuvörderst die nachfolgend zu besprechende Tatsache würdigen. Es ist bereits eine allgemein gültige Tatsache, dass der hungernde Organismus als seine Energiequelle in erster Linie über Kohlehydrate und Fette verfügt und dass erst dann, nachdem sie bis zu einem gewissen Grad aufgebraucht worden sind, die Eiweisstoffe zu diesem Behufe herangezogen werden. Daraus geht also ohne weiteres hervor, dass die Spaltung des Eiweisses im Anfangsstadium des Hungers in geringem Masse vor sich geht, und je länger die Zeit abläuft, um so intensiver die Eiweisszerstzung vonstatten geht.

Was zuerst die Veränderungen der Bluteiweisskörper im kurzfristigen Hungerversuch anbelangt, so vermindert sich Gesamt-N gleichgültig, ob es sich um die absolute Karenz oder um die Wasseraufnahme handelt, während zugleich Rest-N vermehrt, Eiweiss-N vermindert ist. Hieraus dürfte wohl geschlossen werden, dass der Organismus schon bei 4tägiger Karenz das Bluteiweiss zu verbrauchen anfängt, wodurch Abnahmen des Gesamt-N sowie des Eiweiss-N zustande kommen, was verständlicherweise eine Zunahme des Rest- $N$ als des Zersetzungsproduktes des Bluteiweisses zur Folge hat. Aus dem Umstand, dass k. o.D. und Druck pro $100 \mathrm{mg} /$ dl Eiweiss-N gemeinschaftlich herabgesetzt sind, ist wohl anzunehmen, dass das Eiweiss, welches im ersten Stadium des Hungers verbraucht wird, relativ kleinmolkulärer Natur ist, und ferner dass aus den Eiweissvorratsorganen höchstwahrscheinlich verhältnismässig gröbere Eiweissteilchen zum Ersatz für den Eiweissverlust an das Blut abgegeben werden. 
Bei 7tägiger Karenz vermehrt sich im Blut Rest-N sowohl unter der Bedingung, wo Wassertrinken erlaubt wurde, wie auch unter anderweitiger Bedingung in erheblichem Masse, trotzdem Gesamt-N nicht im geringsten abnimmt. Wenn diese Zunahme des Rest- $\mathrm{N}$ durch die Spaltung des Bluteiweisses allein bedingt sein würde, so muissten Gesamt-N und Eiweiss-N gewiss beträchtlich abgenommen haben. In Wirklichkeit aber nimmt Gesamt-N, wie oben geschildert, nicht im geringsten $a b$, Eiweiss- $N$ erfährt höchstens nur eine geringe Abnahme um $3 \%$. Dies spricht offenbar dafür, dass bei 7 tägiger Karenz die Spaltung des Eiweisses vorwiegend in den Geweben intensiv sich vollzieht und Rest- $\mathrm{N}$ als Zersetzungsprodukt desselben in die Blutbahn übertritt, während anderseits der Ersatz des Bluteiweisses von seiten der Eiweissvorratsorgane übernommen wird. Im Hinblick auf die Feststellung, dass hierbei k.o.D. und Druck pro $100 \mathrm{mg} / \mathrm{dl}$ Eiweiss-N annähernd normale Werte beibehalten, dürfte die Annahme wohl gerechtfertigt sein, dass bei derart langfristigem Hunger in den Depotorganen relativ reife Eiweisskörper sich bilden, welche zum Ersatz für verbrauchtes Eiweiss an das Blut angegeben werden. Diese Annahme wird, wie später geschildert, durch die Beobachtung, dass bei langfristiger Karenz die Zunahme des Rest- $\mathrm{N}$ in den Geweben beträchtlich ist, gestützt werden. Ein ähnlicher Befund kann auch aus den von Hatafuku u. Nakazawa ${ }^{9 \text { ) }}$ am Hund erhaltenen Versuchsergebnissen erhoben werden.

In einschlägiger Literatur lauten die Angaben über das Verhalten des Bluteiweisses im Hungerzustand sehr widersprechend. Eine Reihe Autoren (Lewinski, ${ }^{11)}$ Githens, ${ }^{12)}$ Roberts on ${ }^{8)}$ ) behaupten nämlich die Zunahme und andere (Panum, ${ }^{6}$ ) Torbert, ${ }^{13)}$ Bruckman, D'Es o po u. Peter $\mathbf{s}^{\mathbf{1 4})}$ ) sollen das Gegenteil gefunden haben und wieder andere (Salvioli ${ }^{15)}$ und Burckhard $t^{7}$ ) wollen die Stetigkeit des Bluteiweisses gesehen haben. Eine derartige Diskrepanz der Ergebnisse dürfte wohl als eine sekundäre Folge, welche durch die auf Verschiedenheiten der Tierart und der Versuchsmethode beruhende Abweichung der Wechselbeziehung zwischen dem Grad der Bluteindikkung und der Intensität der Eiweisszersetzung verursacht wurde, angesehen werden. Auch über die qualitative Veränderung des Blutei-

11) Lewins ki, Arch. f. d. ges. Physiol., 1903, 100, 611.

12) Githens, Beitr. chem. Physiol. Pathol., 1904, 5, 515.

13) Torbert, Journ. Exp. Med., 1935, 62, 1.

14) Bruckman, $D^{\prime}$ Es o pou. Peters, Journ. Clin. Invest., 1930, 8, 577.

15) Salvioli, Areh. Anat. Physiol, Abt. Physiol., 1881, 269.

16) Tan a ka, Igaku Kenkyu, 1937, 11, 449. 
weisses sind die Meinungen zwischen den Autoren sehr geteilt; Salvioli ${ }^{15)}$ und Tan a $\mathrm{ka}^{16)}$ geben den unveränderten Albumin- Globulinquotienten an, es haben aber Torber $\mathrm{t}^{13)}$ eine Abnahme des Albumins, Burckhardt, ${ }^{7}$ Githen s $^{12}$ ) hingegen eine Zunahme des Globulins nachgewiesen. Immerhin laufen obige Angaben letzten Endes darauf hinaus, dass die Bluteiweissteilchen entweder mehr nach grober Seite hin verschoben werden oder unverändert bleiben, und stimmen mit meiner aus dem Verhalten des k. o. D. sowie Drucks pro $100 \mathrm{mg} / \mathrm{dl}$ Eiweiss-N abgeleiteten Ansicht überein.

Demnächst sei einiges über die Verhältnisse der Eiweisspaltung im Gewebe im Hungerzustand gestreift. Da die Leber als das Zentralorgan für den Stoffwechsel in innigster Beziehung zum Eiweissumsatz steht, möchte hier die Veränderungen in der Leber als Hauptgegenstand der Analysierung betrachten. Bevor man unter Zugrundelegung von den durch meine Bestimmungen gewonnenen Daten über die Bedeutung der in Geweben aufgetretenen Veränderungen der Eiweisskörper eine Betrachtung anstellt, muss man auf folgende zwei Punkte Rücksicht nehmen. Erstens dreht es sich um das Verhalten des Rest-N. Die Ab- und Zunahme des Rest- $\mathrm{N}$ im Blut kann, sofern in der Ausscheidung durch die Niere keine Störung zustande kommt, als eine zuverlässiges Mass für die bestehende oder fehlende Steigerung der Eiweisszersetzung im Organismus dienen; in den Geweben aber sind die Verhältnisse anders, weshalb die Erklärung darüber nicht so schlechthin wie beim Blut abgegeben werden kann. Es kann zwar aus enormer Zunahme des Rest- $\mathrm{N}$ in einem Gewebe eine Steigerung der Eiweisszersetzung im betreffenden Gewebe erschlossen werden, anderseits aber dürfte es gewiss Fälle geben, wo die Zunahme des Rest-N mit der Intensität der Eiweisszersetzung nicht gleichen Schritt hält. Denn der Rest-N-Gehalt im Gewebe hängt im wesentlichen davon ab, ob der im betreffenden Gewebe gebildete Rest-N geschwind von daselbst forttransportiert wird oder nicht. Mit anderen Worten, auch selbst bei ziemlich intensiv erfolgender Bildung des-N sollte die Zunahme des Rest- $\mathrm{N}$ dann relativ geringfügig sein, wenn er unverzüglich in die Blutbahn befördert wird.

Zweitens handelt es sich darum, dass im Hungerversuch die Gewichtsabnahme jedes einzelnen Organes nicht gleichmässig erfolgt. Nach Untersuchungen von Sasakir) sind es im Hungerversuch an Kaninchen von allen Organen die Leber und Milz, deren Gewichtsab-

17) Sas a ki, Jika Zasshi, 1928, Nr. 343, 2158. 
nahme schneller und stärker als die des Körpergewichts zustande kommt; zu denjenigen Organen, deren Gewichtsabnahme mit der des Körpergewichtes nahezu parallel vor sich geht, gehören der Dünndarm und Hoden. Es sollen ferner Niere, Herz, Magen und Ovarium sein, deren Gewichtsabnahme langsamer und leichtergradig als die des Körpergewichtes stattfindet. Auch nach von Tanaka ${ }^{16)}$ im Hungerversuch an Kaninchen erhobenen Daten soll die Leber, wenn auch darunter individuelle Verschiedenheiten vorkommen, nach dem Durchschnittswert aus grossem Material, bei der Karenz von ca. 4 Tagen an Gewicht um ungefähr 15\% verlieren, nach 6tägigem Hunger bis etwa $\frac{1}{2}$ ihres ursprünglichen Gewichtes herabgesetzt sein.

Was die Eiweisswerte in der Leber bei 4tägiger Karenz in vorliegender Untersuchung anbetrifft, nimmt Rest-N im Durchschnitt um $10,8 \% \mathrm{zu}$, Gesamt- $\mathrm{N}$ ist um 6,5\%, Eiweiss- $\mathrm{N}$ um 8,2\% vermindert. Wenn man den Gewichtsverlust der Leber auf ungefähr $15 \%$ schätzt, so kann daraus gefolgert werden, dass sich die Eiweisspaltung in der Leber vollzieht und zugleich das Lebereiweiss in die Blutbahn mobilisiert ist. In anderen Geweben, in Hirn und Niere sind indes die Zunahme des Rest-N weitgehend grösser, indem sie im Hirn 44,6\%, in der Niere $32,8 \%$ beträgt. Gesamt-N nimmt weder im Hirn, noch in der Niere fast ab. Eiweiss- $\mathrm{N}$ ist in der Niere um ein Geringes vermindert, im Hirn aber unverändert.

Dass der Gewichtsverlust der Niere geringgradig ist, ist schon oben angegeben, da das Hirn auf den Stoffwechsel des Organismus direkt keine stoffliche Beziehung hat, erleidet das Hirn im Hungerzustand nur recht geringen Gewichtsverlust. Im Hunger dürfte allerdings die Spaltung des Eiweisses in Hirn, Niere und Leber ebenfalls gesteigert sein, wodurch Rest- $\mathrm{N}$ zunimmt. In der Leber jedoch, gebunden an die Fähigkeit der Leber, die Eiweisstoffe an die Blutbahn abzugeben (dies ist von $\mathrm{Kasugai,}{ }^{18)}$ Horikawa ${ }^{19}$ ) und Yasuda ${ }^{20}$ an hiesiger Klinik experimentell erwiesen worden), müsste Rest- $\mathrm{N}$ prompt in den Blutstrom übertreten, und dementsprechend der Gehalt des Lebergewebes an Rest- $\mathrm{N}$ gegenüber anderen Geweben geringer sein, wobei auch Eiweiss-N des betreffenden Gewebes naturgemäss mehr oder weniger abnehmen dürfte.

Die Erniedrigung des k.o.D. und Drucks pro $100 \mathrm{mg} / \mathrm{dl}$ Eiweiss$\mathrm{N}$ im Hirn deutet höchstwahrscheinlich darauf hin, dass im Hirn die

18) Kasuga i, Tohoku Journ. Exp. Med., 1935, 27, 505.

19) Horikawa, Ibid., 1936, 28, 90.

20) Y a s u d a, Tbid., 1937, 31, 437. 
Zersetzung des Eiweisses vorwiegend die relativ kleinen Eiweissteilchen betrifft. Dass beide Daten in der Niere keine bedeutende Veränderung aufweisen, dürfte wohl dahin gedeutet werden, dass in der Niere sowohl kleine wie auch grosse Eiweissteilchen gleichmässig abgebaut worden sind. Das Absinken des k. o.D. in der Leber dïrfte auf die infolge der Mobilisation ihres Depoteiweisses herbeigeführte Eiweissverminderung zurückzufiuhren sein, und die Stetigkeit des Drucks pro $100 \mathrm{mg} / \mathrm{dl}$ Eiweiss-N in der Leber dürfte sich dahin erklären lassen, dass die Leber ihr Vorratseiweiss als solches ans Blut abgegeben hat. Da Druck pro $100 \mathrm{mg} / \mathrm{dl}$ Eiweiss- $\mathrm{N}$ des Lebereiweisses, welcher durchschnittlich $15,9 \mathrm{~mm} \mathrm{H}_{2} \mathrm{O}$ beträgt, weitgehend niedriger als der des Blutes (uiber $30 \mathrm{~mm} \mathrm{H}_{2} \mathrm{O}$ ) ist, so sinkt auch Druck pro $100 \mathrm{mg} / \mathrm{dl}$ Eiweiss-N des Blutes, wie schon oben geschildert ist, ab.

Was die Werte bei Tieren, denen das Wassertrinken erlaubt wurde, anbelangt, zeigen die Verhältnisse von Gesamt-N, Rest-N und Eiweiss- $\mathrm{N}$ in der Leber keine wesentlichen Abweichungen von denselben bei den die absolute Karenz befolgten Tieren, während das Absinken des k.o.D. und Drucks pro $100 \mathrm{mg} / \mathrm{dl}$ Eiweiss-N aber ziemlich stärker erfolgt. Und der Umstand, dass im Hirn die prozentische $\mathrm{Zu}$ nahme des Rest- $\mathrm{N}$ gering; das Absinken des k.o.D. und Drucks pro $100 \mathrm{mg} / \mathrm{dl}$ Eiweiss-N viel deutlicher ist, dürfte wohl darauf beruhen, dass, obzwar durch Wasserzufuhr die Spaltung der kleinen Eiweissteilchen sich intensiv vonstatten geht, der hierbei entstandene Rest- $\mathrm{N}$ mit Leichtigkeit forttransportiert worden ist. Die Tatsache, dass in der Niere bei annähernder Stetigkeit des Rest-N, Abnahmen des Gesamt$\mathrm{N}$ und des Eiweiss-N und Erhöhungen des k.o.D. sowie Drucks pro 100 mg/dl Eiweiss-N auftreten, spricht meines Erachtens offensichtlich dafür, dass durch Wasserzufuhr relativ gröbere Eiweissteilchen aus der Niere in die Blutbahn mobilisiert worden sind.

Demnächst sei auf die Veränderungen bei langfristiger Karenz, also 7 tägigem Hungerzustand eingegangen. In der Leber nimmt Rest$\mathrm{N}$ um $28,5 \% \mathrm{zu}$, und dennoch zeigt Gesamt-N eine Zunahme um 10,1\%, Eiweiss-N auch eine Zunahme um 8,3\%. Weil in vorliegenden Versuchen an bestimmten Mengen Leberstücken, nicht aber an der ganzen Leber die Gesamteiweissmengen bestimmt worden sind, ist es selbstvertändlicherweise nicht möglich, aus eben geschilderten Zunahmen auf die Vermehrung der Eiweisstoffe in der Leber zu schliessen. Da nach Sa sa ki in) die Kaninchenleber nach 6 . Hungertag die Hälfte ihres ursprünglichen Gewichtes verliert, so muss die Leber, als im ganzen berechnet, ihr Vorratseiweiss in erheblichem Masse ans Blut abgege- 
ben haben. Die auffallende Ermiedrigung des k.o.D. und Drucks pro $100 \mathrm{mg} / \mathrm{dl}$ Eiweiss-N deutet zweifellos auf die Mobilisation der kleinen Vorratseiweisskörper aus der Leber in die Blutbahn hin.

Erhebliche Zunahme des Rest- $\mathrm{N}$ in Hirn und Niere dürfte sich auf dieselbe Weise wie bei 4tägiger Karenz erklären lassen; im Hinblick auf die Feststellung, dass bei unbedeutender Veränderung des GesamtNneben der Abnahme des Eiweiss-N derk.o.D. und Druck pro $100 \mathrm{mg} / \mathrm{dl}$ Eiweiss- $\mathrm{N}$ in erheblichen Masse herabgesetzt sind, ist die Annahme wohl berechtigt, dass auch aus eben genannten Organen relativ feinmolekuläre Eiweissteilchen verschwunden sind.

In den Versuchen, wo das Wassertrinken erlaubt wurde, ist in der Leber die Veränderungen unserer Daten fast nicht nachzuweisen. In Hirn und Niere scheint die Abtransportion des Rest-N mit Leichtigkeit vonstatten zu gehen, so dass dieZunahme des Rest-N weitgehend geringer als bei absoluter Karenz ist. K.o.D. und Druck pro $100 \mathrm{mg} / \mathrm{dl}$ Eiweiss- $\mathrm{N}$ sind in erheblichem Masse erniedrigt, woraus wohl erschlossen werden dürfte, dass die Mobilisation kleiner Eiweissteilchen aus hier in Betracht kommenden Organen durch Wasserzufuhr gefördert wird.

\section{Schluss.}

Die Veränderungen der Eiweisskörper in Blut und Geweben, die in 4tägigem und 7tägigem Hungerversuch an Kaninchen ermittelt worden sind, gestalten sich wie folgt.

1. Veränderungenim Blut.

Hämoglobinkonzentration erfährt infolge der Bluteindickung Erhöhung, die bei absoluter Karenz besonders ausgeprägt ist."

Bezüglich der Eiweisskörper ist bei kurzfristiger Karenz Gesamt$\mathrm{N}$ vermindert, Rest- $\mathrm{N}$ vermehrt, Eiweiss- $\mathrm{N}$ verringert. K.o. D. und Druck pro $100 \mathrm{mg} / \mathrm{dl}$ Eiweiss-N fallen gemeinschaftlich ab. Bei 7 tägiger Karenz nimmt Rest- $\mathrm{N}$ beträchtlich zu, wobei Gesamt-N keine Abnahme aufweist, Eiweiss-N nimmt aber gewissermassen ab. Kolloid-osmotischer Druck und Druck pro $100 \mathrm{mg} / \mathrm{dl}$ Eiweiss-N behalten annähernde Normalwerte bei.

2. Veränderungen in Geweben(Leber, Hirn und Niere). Leber : Bei 4tägiger Karenz nimmt Rest-N zu, während Gesamt$\mathrm{N}$ und Eiweiss-N abnehmen. Kolloid-osmotischer Druck sinkt ein wenig ab, Druck pro $100 \mathrm{mg} / \mathrm{dl}$ Eiweiss-N erfährt aber keine wesentliche Veränderung. 
Bei 7tägiger Karenz weist Rest- $N$ noch erheblichere Zunahme auf, und dennoch zeigt sowohl Gesamt-N wie auch Eiweiss-N eine wenn auch geringfügige Zunahme. Hierbei erniedrigen sich der kolloid-osmotische Druck und Drúck pro $100 \mathrm{mg} / \mathrm{dl}$ Eiweiss-N in beträchtlichem Masse.

Hirn : Bei 4tägiger Karenz nimmt Rest-N auffallend zu, Gesamt$\mathrm{N}$ und Eiweiss- $\mathrm{N}$ nehmen aber nicht ab. Nichtsdestoweniger fällt der kolloid-osmotische Druck ausgeprägt herab. Bei 7tägiger Karenz tritt die Zunahme des Rest- $\mathrm{N}$ noch stärker zutage als bei 4 tägiger $\mathrm{Ka}$ renz, Eiweiss-N nimmt ab, ohne dass jedoch Gesamt-N verringert ist ; das Absinken des kolloid-osmotischen Drucks und Drucks pro 100 $\mathrm{mg} / \mathrm{dl}$ Eiweiss- $\mathrm{N}$ ist hier ausserordentlich erheblicher als bei 4 tägiger Karenz.

Niere : Bei 4tägiger Karenz vermehrt sich Rest- $N$ in beträchtlichem Masse, Gesamt-N erfährt kaum nennenswerte Veränderung. Eiweiss- $\mathrm{N}$ ist mehr weniger vermindert, der kolloid-osmotische Druck und Druck pro $100 \mathrm{mg} / \mathrm{dl}$ Eiweiss-N bieten keine wesentlichen Veränderungen dar. Bei 7tägiger Karenz nimmt Rest-N zwar ausgesprochen zu, wogegen Gesamt-N fast keine Veränderung aufweist ; und bei geringer Abnahme des Eiweiss- $\mathrm{N}$ erniedrigen sich der kolloid-osmotische Druck und Druck pro $100 \mathrm{mg} / \mathrm{dl}$ Eiweiss-N:

3. Aus dem oben Gesagten geht klar hervor, dass bei zweierlei Karenzen (kurz- und langfristiger) in Versuchen, wo Wasser freiwillig genommen worden ist, die Zunahme des Rest- $\mathrm{N}$ im allgemeinen in gewissermassen geringerem Grad erfolgt, wogegen die Erniedrigung des kolloid-osmotischen Drucks sowie Drucks pro $100 \mathrm{mg} / \mathrm{dl}$ Eiweiss$\mathrm{N}$ etwas grösser ist. 